\title{
BEYOND IMMUNIZATION: TRAVELERS' INFECTIOUS DISEASES 1- DIARRHEA \\ By
}

\author{
MAMDOUH M. M. EL-BAHNASAWY AND TOSSON A. MORSY \\ Consultant of Endemic Diseases and Fevers, Military Medical Academy and Depart- \\ ment of Parasitology, Faculty of Medicine, Ain Shams University, Cairo 11566, Egypt
}

\section{Abstract}

Travelers' diarrhea is the most common illness in persons traveling from resource-rich to resource-poor regions of the world. The fear of developing diarrhea while traveling is common among travelers to any part of the developing world. This concern is realistic; 40 to $60 \%$ of travelers to these countries may develop diarrhea. Diarrheal diseases represent one of the five leading causes of death worldwide. Morbidity and mortality are significant even in the United States where diarrhea is more often than not a "nuisance disease" in the normally healthy individual.

Key words: Travelers, Bacteria, Virus, Parasites, Foodborne, Diarrhea.

\section{Review and Discussion}

Episodes of travelers' diarrhea (TD) are nearly always benign and self-limited, but the dehydration that can complicate an episode may be severe and pose a greater health hazard than the illness itself. Nevertheless, it is possible to educate a traveler to manage a diarrheal episode without compromising either their trip or their health (Hill et al, 2006).

The following definitions were suggested according to the duration of diarrhea: acute$\leq 14$ days in duration, persistent diarrheamore than 14 days in duration and chronicmore than 30 days in duration. Cases of acute diarrhea are due to infections with viruses and bacteria and are self-limited. Noninfectious etiologies become more common as the course of the diarrhea persists and becomes chronic. The evaluation of patients for a noninfectious etiology should be considered in those patients in whom evaluation fails to identify a pathogen (e.g., bacterial, viral, or protozoan) and the diarrhea worsens or becomes chronic (Musher and Musher, 2004).

Travelers' diarrhea (TD) is frequently categorized into three forms: classic, moderate, and mild. For epidemiologic studies, the three types can be grouped together to estimate a total number of cases of TD. These forms of TD are defined as follows: Classic- passage of three or more unformed stools in a 24 hour period plus at least one of these other symptoms are nausea, vomiting, abdominal pain or cramps, fever, blood in stools Moderate-passage of one or two unformed stools in 24 hours plus at least one of the above symptoms or more than two unformed stools in 24 hours without other symptoms Mild- passage of one or two unformed stools in 24 hours without other symptoms (von Sonnenburg et al, 2000)

These definitions allow some uniformity in studies of the epidemiology and etiologies of TD. TD should also be in the differential diagnosis when diarrhea develops within 10 days after the individual returns home.

Epidemiology: Diarrheal disease in travelers may be caused by a variety of bacterial, viral, and parasitic organisms, which are most often transmitted by food and water (Steffen, 1986). More than $90 \%$ of illnesses in most geographic areas are caused by bacteria; the most common organisms are Salmonella, Campylobacter, Shigella, Escherichia coli 0157:H7 \& Clostridium difficile (DuPont and Capsuto, 1996). In one series of 30,369 travelers to Jamaica, the attack rate for TD was $24 \% ; 12 \%$ of these cases were the classic form. Departing travelers surveyed at the airport reported incapacity for a mean of 11.6 hours from the illness; fewer than three percent stated that they had 
attempted to avoid potentially high-risk foods or drinks (Mattila et al, 1992).

The epidemiology of TD does vary from location to location and with the season of the year. Spices in food and changes in climate do not cause TD, although variations in diet, temperature, or even time zones can alter the way a traveler feels and the stresses of travel may exacerbate diarrheal symptoms (Steffen et al, 1999).

The world can be divided into three regions by risk for the development of TD: Low risk $(<10 \%)$ - Northern Europe, Australia and New Zealand, the United States, Canada, Singapore, Japan Moderate risk (10 to $20 \%$ ) - Caribbean Islands, South Africa, and countries bordering the Mediterranean Ocean including Israel High risk $(>30 \%)$ Asia (with the exception of Singapore), Africa (outside of South Africa), South and Central America, and Mexico

Travelers should be aware that food items on aircraft will often be obtained at the city of departure (CDC, 1971)

Risk factors: The development of diarrhea is related to the number of ingested organisms that reach the intestine alive. Thus, any factor which enhances the ability of bacteria to survive ingestion and transit to the intestine will increase the risk for the development of diarrheal disease. As an example, an individual who is taking histamine blockers for ulcer disease will be at increased risk for developing diarrhea since the reduction of gastric acid will allow ingested pathogens prior to their entry into the small bowel. Similarly, an individual who has altered upper gastrointestinal (GI) anatomy (eg, after ulcer surgery, or with a blind loop syndrome) or motility may be at increased risk for the development of diarrheal disease while traveling in more contaminated environments. However, it is not clear what factors beyond exposure may influence the acquisition of parasites or viruses (Soave and Ma, 1985).

Ingestion of parasites that cause diarrhea requires a more contaminated environment than is usually frequented by the average traveler. Thus, parasitic pathogens rarely cause TD. However, there are a few locations where travelers are more likely to acquire parasites, including Nepal (where both Giardia lamblia and Cyclospora cayetanensis are common) and St. Petersburg (where G. lamblia remain hyperendemic). The mountainous regions of the West and Northeast United States are also highly endemic for $G$. lamblia, but travelers to these locations rarely request advice prior to travel. In these situations, it may be that environmental factors such as the juxtaposition of the water supply with the habitat of a certain animal species predispose the area to a hyper-infestation with the parasite (Jokipii et al, 1985).

1- Bacteria: Bacterial pathogens predominate as the cause of TD. In the series of 322 patients cited above, ETEC accounted for 12 percent of cases due to bacteria followed by Salmonella spp., Campylobacter jejuni, and Shigella spp. at 8, 6, and $0.3 \%$, respectively. In another study of 636 travelers to Guadalajara Mexico, Coho Rios Jamaica, and Goa India, the more newly described enter aggregative E. coli (EAEC) were responsible for $26 \%$ of cases, second only to ETEC with $30 \%$. The identification of EAEC in these stool specimens reduced the number of cases with an undiagnosed etiology from 51 to $37 \%$.

Other organisms (Salmonella spp, Shigella spp, C. jejuni, Vibrio spp., Aeromonas hydrophila, Plesiomonas shigelloides as well as rotavirus and the parasites Entamoeba histolytica, Cryptosporidium parvum, and G. lamblia) accounted for $7 \%$ of cases. Twenty percent also had infection with more than one enteric organism (Adachi et al, 2001).

Patients taking malaria prophylaxis or other antibiotics may also occasionally develop antibiotic-associated diarrhea due to Clostridium difficile, but not a common cause of TD (Golledge and Riley, 1995).

2- Viruses: Viruses can also cause TD as Calciviruses (Norovirus and related viruses), Rotavirus, Adenovirus types 40 and 41 \& 
Astrovirus. Rotaviruses are the most common among the viral pathogens, accounting for $9 \%$ of cases in the series from Jamaica. It is not always clear that the individual acquires the virus while traveling rather than incubating the infection prior to departure, with symptoms developing during the trip.

3- Parasites: Although parasitic travelers' diarrhea may be rare, Cryptosporidium parvum, Microsporidia, and Isospora belli, in addition to Giardia lamblia and Cyclospora cayetanensis, may contribute to diarrhea in travelers. Entameba histolytica can produce intestinal infection. Other parasites, such as Ascaris lumbricoides or Strongyloides stercoralis, are not usually associated with diarrheal symptoms (el-Karamany et al, 2005).

Clinical Manifestations: A diagnostically important finding is fever, which suggests infection with invasive bacteria e.g., Salmonella, Shigella, or Campylobacter (Burchard et al, 2013), enteric viruses, or a cytotoxic organism such as Clostridium difficile (Koo and DuPont, 2010) or Entamoeba histolytica even caused hepatopulmonary amoebiasis with infectious phlebitis (Patrício et al, 2014). Blastocystis hominis was associated with travelers' diarrhea and responded to iodoquinol or metronidazole (Markell and Udkow, 1986).

Endoscopy - Endoscopy is uncommonly needed in the diagnosis of acute diarrhea. It may be helpful in the following settings: Distinguishing inflammatory bowel disease from infectious diarrhea. Distinction between Crohn's disease of the colon-rectum and ulcerative colitis or inflammatory bowel disease (IBD) type unclassified can be of pivotal importance for a tailored clinical management, as each entity often involves specific therapeutic strategies and prognosis. Novel advanced endoscopic imaging techniques and biomarkers can shed new light for the differential diagnosis of IBD, better reflecting diverse disease behaviors based on specific pathogenic pathways. (Tontini et al, 2015). Clostridium difficile infection has increased in incidence and severity over the past quarter century, and now considered a major cause of healthcare-associated infections. Diagnosing $C$. difficile infection and looking for pseudo-membranes in the patients who are toxic while results of tissue culture assays are pending. The widespread adoption of ELISA for $C$. difficile toxins A and $\mathrm{B}$ has reduced the time for $C$. difficile results to become available and thus decreased the need for endoscopy in these patients (To and Napolitano, 2014). In immunocompromised patients who are at risk for opportunistic infections with agents such as cytomegalovirus. In patients in whom ischemic colitis is suspected but the diagnosis remains unclear after clinical and radiologic assessment. Chronic constipation, entericcoated aspirin intake and splanchnic atherosclerosis are risk factors related to ulcer in ischemic colitis (IC) patients. Also, the abdomen tenderness, high $\mathrm{WBC}$ and low $\mathrm{Hb}$ strongly indicate possible IC with ulcer (Liu et al, 2014). Wang and $\mathrm{Xu}$ (2012) investigated the clinical manifestations, pathological features by endoscopy and possible risk factors ofischemic colitis (IC).IC symptoms are not typical, it requires early colonoscopy to clarify diagnosis. Hypertension, diabetes, hyperlipidemia, and atrial fibrillation are risk factors for IC. Moreover, the gastrointestinal aspergillosis is an unusual presentation of invasive Aspergillus associated with a high mortality rate. Characteristic features of gastrointestinal aspergillosis include invasion of the mesenteric arteries, intravascular thrombosis and subsequent tissue ischemia. Clinical manifestations of invasive Aspergillus of the gastrointestinal tract can include fever, abdominal pain, ileus, peritonitis, bloody diarrhea or hematochezia. In an autopsy series of patients with invasive Aspergillus, 37 of 107 patients had Aspergillus involvement of the gastrointestinal system; the commonest pathological findings included ulcers and abscesses. Although rare, invasive aspergillosis may present with gastrointestinal bleeding associated with necrotic 
ulcers on endoscopic examination (Bizet et al, 2014).

Most clinicians consider a foodborne illness when a patient presents with gastrointestinal symptoms including nausea, vomiting, abdominal pain, diarrhea and fever. However, other manifestations of foodborne illness can occur, such as neurologic symptoms (e.g., headaches, paralysis or tingling), hepatitis, and renal failure. Some foodborne agents, such as C. botulinum, L. monocytogenes, or $V$. vulnificus, can be rapidly lifethreatening and speedy therapy may be lifesaving. Maintaining an open mind in relation to the plethora of presentations of foodborne disease and remembering to ask about food exposures are both key elements in the diagnosis of foodborne diseases. A food history may also provide clues to a diagnosis. Consumption of unpasteurized dairy products, raw or undercooked meat or fish, or organic vitamin preparations may suggest certain pathogens. In addition, the timing of symptoms with regard to exposure to suspected offending food can be important clues to the diagnosis. Women who are pregnant have a 20 -fold increased risk of developing listeriosis from meat products or unpasteurized dairy products such as the soft cheeses or raw fish (El-Bahnasawy et al, 2014). There are no simple algorithms to diagnose foodborne illness. However, there are some key factors that will point the physician in the right diagnostic direction. Three key questions reviewed here: What are the probable microbial causes of foodborne disease? How do time course and types of symptoms serve as clues? How can a food history help to narrow the diagnosis? In order to make a diagnosis of foodborne disease, one first has to consider the spectrum of clinical manifestations (Helms et al, 2006). A foodborne disease will typically manifest as a mixture of nausea, vomiting, fever, abdominal pain and diarrhea. However, some foodborne diseases may not have gastrointestinal symptoms. As examples, botulism and some types of shellfish poisoning can present with pa- ralysis, ciguatera fish poisoning or scombroid with headaches and tingling, amnesic shellfish poisoning with amnesia, hepatitis A or E with hepatitis and Listeria monocytogenes with meningitis or spontaneous abortion (Alonso et al, 2015).

Besides, there are three important elements of the food history that the physician should consider while trying to determine the differential diagnosis of foodborne diseases: apresenting symptoms, b- exposure to a particular type of food associated with foodborne disease and c- time interval between exposure to the suspect food and the onset of symptoms (Varma et al, 2012)

The symptoms that begin within six hours suggested the ingestion of a preformed toxin of Staphylococcus aureus or Bacillus cereus, symptoms that begin at 8 to 16 hours suggest infection with Clostridium perfringens, and symptoms that begin at more than 16 hours can result from viral or bacterial infection (e.g., contamination of food with enterotoxigenic or enterohemorrhagic $E$. coli). Syndromes that may begin with diarrhea but progress to fever and more systemic complaints such as head ache, muscle aches, stiff neck may suggest infection with Listeria monocytogenes, particularly in pregnant woman. Most episodes of TD occur between 4 and 14 days after arrival, but can occur within a much shorter time frame if the concentration of bacteria ingested is sufficiently high. The illness is generally self-limited with symptoms lasting for approximately one to five days. However, 8 to $15 \%$ of patients experience symptoms for more than one week and as many as two percent for more than one month. While it is common to be unable to proceed with planned activities, only approximately $20 \%$ of patients report requiring bed-rest for one to two days.

The symptoms of TD depend upon the microbial etiology. The classic "turista" due to ETEC generally produces malaise, anorexia, and abdominal cramps followed by the sudden onset of watery diarrhea. Nausea and vomiting also may occur. Typically there are 
no symptoms of colitis such as blood or pus in the stool. Patients may develop a low grade fever (Rendi-Wagner and Kollaritsch, 2002).

Even when other bacterial agents such as C. jejuni and Shigella spp. are implicated, the symptoms initially experienced by the traveler may be similar to those seen with ETEC. However, infections with these organisms may progress to include symptoms of colitis, such as tenesmus, urgency, cramping and bloody diarrhea.

Belching and other upper intestinal symptoms are typical of giardiasis, while profuse watery diarrhea is characteristic of cryptosporidiosis and $C$. cayetanensis infection. The symptoms of microsporidiosis may be more subtle with bloating and intermittent diarrhea.

Diagnosis: Indications for diagnostic evaluation in patients with relatively severe illness, as suggested by one or more of the following: 1-Profuse watery diarrhea with signs of hypovolemia 2- Passage of many small volume stools containing blood and mucus. 3- Bloody diarrhea. 4-Temperature $\geq 38.5^{\circ} \mathrm{C}$ $\left(=101.3^{\circ} \mathrm{F}\right)$. 5- Passage of $\geq 6$ unformed stools per 24 hours or a duration of illness $>48$ hours. 6- Severe abdominal pain 7- Recent use of antibiotics or hospitalized patients. 8- Diarrhea in elderly $(\geq 70$ years of age) or immunocompromised. \& 9- Systemic illness with diarrhea, especially in pregnant women, which case listeriosis should be suspected (Thielman and Guerrant, 2004)

Since TD is generally self-limited, treatment is often symptomatic and initiated without documenting an etiologic agent. However, if symptoms are severe and associated with toxicity or if they persist beyond 48 to 72 hours, intervention may be necessary. Routine stool cultures are rarely warranted routine since ETEC and EAEC cannot be distinguished from nonpathogenic $E$. coli on stool culture and viral agents would not be identified with stool cultures. A stool culture should be sent in a patient with fever and colitis symptoms. In patients with pre- dominantly upper GI symptoms (e.g., bloating, gas, nausea), stool examination for $G$. lamblia and Cyclospora should be undertaken. The itinerary of the traveler should be considered when deciding which patients to culture or obtain special stains for parasites. Examination for C. parvum, Microsporidi$u m$, or other less common organisms should only be initiated when diarrhea has persisted for more than 10 to 14 days.

Treatment: The treatment of TD may include three different modalities: Fluid replacement, antibiotics and anti-motility agents

Fluid replacement: Fluid replacement is essential; antibiotics and anti-motility agents may be required depending upon the circumstances. Most cases are self-limited and resolve on their own within three to five days of treatment with fluid replacement only. Antimicrobial therapy shortens the disease duration to about one day and anti- motility agents may limit symptoms to a period of hours.

The primary and most important treatment of travelers' (or any other) diarrhea is fluid replacement since the most significant risk is volume depletion. Patients with mild diarrhea may combine alternating sips of fluids with both salt and sugar to replete and maintain hydration. Broth, fruit juice, or similar fluids may be used. Pedialyte is frequently useful in children.

Severe diarrhea should be treated with oral rehydration solution; this replaces needed electrolytes in the appropriate concentrations. These solutions were developed following the realization that intestinal glucose linked sodium absorption remains intact in most small bowel diarrheal illnesses. Thus, in diarrheal disease caused by any organism that activates small bowel secretory processes (eg, cholera toxin turning on cAMP), the intestine remains able to absorb water if glucose and salt are also present to assist in the transport of water from the intestinal lumen (Avery and Snyder, 1990). 
Packets of oral rehydration solution are available in the pharmacies of most countries and can be mixed with clean drinking water. Alternatively, a similar solution can be made by adding $1 / 2$ teaspoon of salt, $1 / 2$ teaspoon of baking soda, and 4 tablespoons of sugar to one liter of water. The electrolyte concentrations of fluids used for sweat replacement (eg, Gatorade) are not equivalent. If available, racecadotril, an enkephalinase inhibitor, may be an effective adjunct to oral rehydration solutions.

For mild diarrhea, the use of fluids is the critical factor; the fluid need not be oral rehydration solution. One study showed no difference in outcome between treatment with the oral rehydration solution plus the loperamide versus generic fluids and the loperamide (Salazar-Lindo et al, 2000).

Diet: The optimal dietary intake has not been resolved. Controversy exists about such issues as partial fasting, the composition of the diet, and the time at which solid food intake should be resumed. A restricted diet (e.g., beginning with only clear liquids to match diarrheal losses during the acute phase of diarrhea) is often recommended.

A pilot randomized trial compared the effects of this restricted diet to an unrestricted diet in which the only specific recommendation was to match fluid intake to diarrheal losses. The study subjects were healthy American college students being treated with an antimicrobial agent in Mexico. The mean duration of diarrhea (37 versus 33 hours) and the course of clinical symptoms were similar in the two groups (Huang et al, 2004).

The general applicability of these observations is uncertain. Furthermore, diet other than hydration is not likely to be important since disease duration is only about one to two days with antibiotic therapy.

Antibiotics: Antibiotics are warranted to treat diarrhea in those who develop moderate to severe diarrhea as characterized by more than four unformed stools daily, fever, blood, pus, or mucus in the stool. In addi- tion, some travelers desire antibiotic treatment for milder disease if the illness is a large burden on a business trip or vacation. Travelers may be given a prescription for antibiotics that can be taken if diarrhea develops rather than as prophylaxis.

Travelers generally should medicate themselves rather than seek medical advice while traveling. However, medical help may be needed in patients who develop high fever, abdominal pain, bloody diarrhea, or vomiting and empiric antibiotics have not been of benefit. For most patients while traveling or after returning home, medical consultation is not needed unless symptoms persist for 10 to 14 days.

Quinolones: When antibiotics are indicated, therapy with a quinolone antibiotic can be initiated after the diarrhea begins. Most commonly, ciprofloxacin (500 mg twice daily) is given for one or two days, although any of the newer, once a day quinolones should be effective. Quinolones are not approved for the pregnant women or children.

The quinolones will lead to resolution of diarrheal symptoms in the majority of travelers within one day (Taylor et al, 1991). In two randomized trials, for example, ciprofloxacin (500 $\mathrm{mg}$ twice daily) resulted in a mean duration of diarrhea of 1.5 days compared to 2.9 days with placebo and norfloxacin (400 mg twice daily for three days) resulted in a mean duration of diarrhea of 1.2 days compared to 3.3 days with placebo Although two to three days should be sufficient for the majority of episodes of TD, a single dose of ciprofloxacin or norfloxacin may also be effective (Salam et al, 1994).

All of the quinolones can be expected to have similar activity against the organisms that cause TD; the most readily available, easiest to tolerate, and most inexpensive quinolone should be prescribed. When ciprofloxacin is used by an individual who drinks caffeine containing beverages, the drug may increase caffeine levels and cause jitteriness. The newer once daily quinolones (e.g., levofloxacin and moxifloxacin) would 
be expected to be active but have not been approved for use in infectious diarrhea.

The quinolones are active against the majority of ETEC strains and also have activity against less common but potential pathogens such as Campylobacter spp, Salmonella spp, $V$. parahaemolyticus, and, although rarely a cause of TD, Vibrio cholerae (CDC, 1992). One concern is the increasingly frequent resistance to quinolones among diarrheal pathogens worldwide, particularly Campylobacter jejuni isolates in Southeast Asia. For this reason, azithromycin is an increasingly common alternative to a quinolone, particularly in travelers to Asia (Smith et al, 1999).

Azithromycin: Azithromycin is an effective drug for the treatment of TD and evidence of efficacy has been provided by studies from Mexico, Turkey, and Thailand (Hakanen et al, 2003). In randomized controlled trials of American adults with TD in Mexico and Turkey, a single $1000 \mathrm{mg}$ oral dose of azithromycin was as effective as a single $500 \mathrm{mg}$ dose of levofloxacin. In the trial from Turkey, azithromycin was associated with a greater likelihood of nausea than levofloxacin in the first 30 minutes after dosing, 8 versus 1\% (Adachi et al, 2003).

Azithromycin may have a particular role in the treatment of travelers' diarrhea in Southeast Asia where, as noted in the previous section, quinolone-resistant Campylobacter jejuni is a common cause. A randomized trial performed in Thailand compared azithromycin, given as a single $1 \mathrm{~g}$ dose or $500 \mathrm{mg} /$ day for three days, with levofloxacin (500 mg/day for 3 days) in 156 United States military personnel. C. jejuni was responsible for 64 percent of cases and was quinolone-resistant in 50\% (Sanders et al, 2007).

Azithromycin administered as a single 1-g dose had a higher cure rate than a 3-day regimen of either azithromycin (500 mg daily) or levofloxacin (500 mg daily); cure rates were 96,85 and $71 \%$, respectively. Microbiologic eradication was much higher with azithromycin (96 to $100 \%$ versus $38 \%$ with levofloxacin), but this difference correlated poorly with outcome.

Rifaximin: Rifaximin (200 mg three times daily for three days) is a nonabsorbed rifamycin that has been demonstrated to be effective in the treatment of TD caused by noninvasive strains of $\mathrm{E}$. coli. It is attracting increasing interest because of concerns about quinolone resistance (Steffen et al, 2003).

In controlled trials, rifaximin was associated with more rapid cessation of diarrhea than placebo and had equal efficacy to ciprofloxacin. Rifaximin combined with loperamide may provide more rapid symptomatic improvement than either agent alone (Dupont et al, 2007).

Resistance: Widespread antibiotic resistance to drugs (such as ampicillin and trimethoprim-sulfamethoxazole) has made these agents less useful for the treatment of TD in much of the world. Resistance has developed among many routine enteric pathogens. In addition, organisms such as Campylobacter spp or any of the vibrios would not be expected to be sensitive to these agents (Murray, 1986).

Treatment approach: The patient should be given enough medication for three days of therapy (except for azithromycin which is $1000 \mathrm{mg}$ as a single dose). The treatment may be discontinued after 24 hours if symptoms have improved (Murray et al, 1990).

Other agents: Bismuth subsalicylate can also be used to treat diarrhea although large doses are required. Sixty $\mathrm{mL}$ (or four tablets) should be taken every one-half hour until the diarrhea resolves or eight doses have been taken. The two major disadvantages of this type of treatment are the potential for salicylate toxicity (especially in those who take aspirin for any reason, pregnant women, and children) and the need to carry large quantities of bismuth subsalicylate.

An antisecretory agent derived from plants (SP-303 [Provir]) was tested in a doubleblind placebo-controlled study of TD in 184 
travelers from the United States to Jamaica or Mexico. The duration of TD was reduced by $21 \%$ among those taking SP-303, and the incidence of treatment failure was significantly decreased compared to placebo. This may be another promising approach (DiCesare et al, 2002).

Anti-motility agents: Anti-motility agents such as loperamide (Imodium) or diphenoxylate (Lomotil) are frequently used by travelers in combination with antibiotics to reduce the rate of stooling; they do not treat the cause of diarrhea. The symptomatic improvement associated with their use in this setting has been somewhat limited but considered to be of benefit. A 2008 metaanalysis of 12 studies suggested that the loperamide combined with antibiotic therapy is beneficial to the traveler with diarrhea (Petruccelli et al, 1992).

In one study, for example, 104 patients with TD were treated with ciprofloxacin 500 $\mathrm{mg}$ twice daily for three days. They were randomly assigned to receive loperamide (4 $\mathrm{mg}$ first dose and $2 \mathrm{mg}$ for every loose stool up to $16 \mathrm{mg} /$ day) or placebo, in addition to the ciprofloxacin. After 24 hours, symptoms had improved or fully recovered in $82 \%$ of the loperamide group compared with $67 \%$ of those receiving placebo. After 48 hours, the symptoms of $90 \%$ of both groups had improved or fully recovered (Riddle et al, 2008).

There continues to be some concern that anti-motility agents can prolong some types of dysenteric illnesses as, Shigella. While some studies suggest that anti-motility drugs can be safely used in dysenteric illnesses as long as they are combined with antibiotic therapy, the studies included in the 2008 meta-analysis all excluded patients with bloody diarrhea or symptoms suggestive of dysentery. Thus, caution should be exercised in using these agents in travelers with bloody diarrhea (DuPont and Hornick, 2003).

Travelers often elect to take anti-motility agents in certain circumstances (eg, a prolonged bus or car trip). Particular vigilance about hydration is important in these patients since the anti-motility drugs do not kill the pathogen causing the diarrhea or stop the secretory process in the intestine. Patients may be unaware of how much fluid they are losing into their intestine since they are no longer having frequent bowel movements.

Recommendation: Cautious is a must in use of anti-motility agents in the setting of TD. They should be administered only in conjunction with empiric antibiotic treatment of the offending diarrheal pathogen, and travelers should be educated about the need to avoid usage in dysentery. Antimotility agents should be stopped if abdominal pain or other symptoms worsen or if the diarrhea continues to be intractable after two days.

Prevention: There are several means by which travelers can reduce their risk of developing a diarrheal illness, including the following: Improving food and drink selection water purification prophylactic medications

Food and drink selection: Educated choices in selecting food and drinks can result in a lower incidence of diarrheal disease. Travelers should be aware of the following observations regarding transmission of diarrheacausing organisms: Freezing does not kill the organisms that cause diarrheal disease. Thus, ice in drinks is not safe unless made from adequately boiled or filtered water. Alcohol does not sterilize water or ice; mixed drinks may still be contaminated. Outbreaks of diarrheal disease have been associated with bottled water (including carbonated water with insufficient carbonation) on rare occasions (Ericsson et al, 1980). However, the presence of carbonation when a bottle is opened (eg, carbonated water or soft drinks) can reassure the traveler that the drink was processed in a proper fashion and is usually safe. Fruit salads, lettuce, or chicken salads are examples of unwise food choices; the ingredients may have been improperly washed and/or may have been sitting for some time without proper refrigeration. 
Condiments on the table can frequently become contaminated. One study of Mexican sauces in restaurants in Guadalajara and Houston found $E$. coli in more sauces in Mexico than in Houston (66 versus $40 \%$ ); it is important to note that significant levels of contamination was not limited to Mexico (Adachi et al, 2002). Guacamole was the most frequently contaminated and was second only to pico de gallo for the highest colony count of bacteria. Steam table buffets that offer the traveler a variety of foods from the local region are risky since the temperature of the food can promote the growth of bacteria.

Hot tea and coffee are usually safe alternatives to boiled water. Bottled drinks should be requested without ice and should be drunk from the bottle with a straw rather than from a glass. Fruits that can be peeled are safe as long as they are peeled just prior to eating (DuPont et al, 2005).

Water purification: It is usually safe to assume that the traveler will be able to find bottled water or soft drinks unless travel is to a rather remote area. Travelers who are going to be living in rustic circumstances overseas will need to make arrangements for a safe water supply depending upon their circumstances. Water can be purified in one of several ways: Boiling for 3 minutes followed by cooling to room temperature (do not add ice) to kill bacteria, parasites, and viruses Adding two drops of 5 percent sodium hypochlorite (bleach) to a quart of water will kill most bacteria in 30 minutes Adding five drops of tincture of iodine to a quart of water will kill bacteria within 30 minutes Compact water filters in which the filters are impregnated with iodine remove parasitic pathogens and kill viral and bacterial pathogens; they provide a reasonable alternative for those who expect to be traveling under rustic circumstances. These are available commercially at camping or wilderness supply stores (Hilton et al, 1997).

The boiling water is usually the most pala- table solution to water purification if sanitary storage is feasible. The addition of iodine or chlorine to water can impart an unpleasant taste. Groh et al. (1996) reported that for travelers or campers, water being safe to drink if heated to boiling, but heating water until it was "too hot to touch" was inadequate for safe drinking purposes.

Prophylaxis: Both antibiotics and certain other drugs have been evaluated for the prevention of TD.

Antibiotics: Prophylactic antibiotics prevent the majority of diarrheal disease in travelers, but cannot be recommended unless the complications of diarrhea or an underlying medical condition make the consequence of dehydration so severe that the benefits of using antibiotic prophylaxis outweigh the risks. Daily antibiotics are expensive and have potential side effects that may exact a medical cost that is unacceptable. The side effects include sun sensitivity, allergic reactions, altered GI flora with colonization by resistant bacteria, yeast infections such as candidal vaginitis, and the risk of $C$. difficile colitis.

Some situations in which it might be reasonable to consider prophylactic antibiotics include: known severe inflammatory bowel disease which could be exacerbated by an episode of infectious diarrhea; severe vascular, cardiac, or renal disease that would be seriously compromised by dehydration; or severe immunocompromise such as advanced HIV disease or after a complicated organ transplant such as a liver or cardiac transplant. Studies with prophylactic antibiotics were predominantly performed with older agents such as TMP-SMX or doxycycline. However, these drugs are generally not used since susceptibility among the bacteria causing TD has changed.

Most travelers requiring prophylaxis are given a quinolone antibiotic at the same doses used for treatment. A number of studies, particularly with norfloxacin, showed that quinolones had a protective efficacy of 80 to $100 \%$. However, emerging resistance among 
pathogens, particularly Campylobacter spp. to the quinolones, especially in Southeast Asia, raise questions about how long prophylaxis with these drugs continues to be effective.

Concerns regarding the development of fluoroquinolone resistance have led to increased interest in the use of rifaximin, which is not absorbed. In a randomized, double-blind placebo-controlled trial in Mexico, 210 American adults received either rifaximin (doses included $200 \mathrm{mg}$ daily, $200 \mathrm{mg}$ twice daily, or $200 \mathrm{mg}$ three times daily) or placebo for two weeks. The following findings were noted: There was a significant reduction in TD with rifaximin therapy (15 versus 54\% with placebo). Protection from illness was similar at all rifaximin doses. The rates of adverse events were comparable in all treatment and placebo arms. Rifaximin therapy was associated with minimal changes in intestinal flora (Briand et al, 2006).

Oral agents for self-treatment of travelers' diarrhea (Hill et al, 2006): 1- Norfloxacin $400 \mathrm{mg}$ twice daily for up to three days, but not recommended. 2- Ciprofloxacin 500mg twice daily for up to three days 20 to 30 $\mathrm{mg} / \mathrm{kg}$ per day in two divided doses for up to three days; maximum dose 500mg. 3- Ofloxacin $200 \mathrm{mg}$ twice daily for up to three days $7.5 \mathrm{mg} / \mathrm{kg}$ every 12 hours for up to three days; maximum dose 200mg. 4- Levofloxacin $500 \mathrm{mg}$ once daily for up to three days $10 \mathrm{mg} / \mathrm{kg}$ once daily for up to three days; maximum dose 500mg, 5- Azithromycin $§$ $1000 \mathrm{mg}$ single dose $10 \mathrm{mg} / \mathrm{kg}$ once daily (single dose); maximum dose $1000 \mathrm{mg}$. 6Rifaxamin 200mg three times daily for up to three days 12 years: $200 \mathrm{mg}$ three times daily for up to three days. Nevertheless, the selftreatment of travelers' diarrhea in children is controversial (Christenson, 2008).

Other agents: Non-antibiotic preventive as Bismuth subsalicylate $(30 \mathrm{~mL}$ or two tablets four times daily with meals) can prevent a significant number of cases of TD. However, the doses required are inconvenient for the traveler, and the same cautions about salicylate toxicity apply when used for prevention as well as therapy.

Probiotics such as Lactobacillus GG have been shown to decrease the incidence of diarrhea in travelers in randomized controlled trials. However, another lactobacillus preparation, nonviable Lactobacillus acidophilus (LA) showed no beneficial effect compared to placebo in a randomized, double-blind, controlled trial in 174 travelers (Clemens et $a l, 1988)$. The authors speculate that the lack of benefit compared to that seen with Lactobacillus GG may be due to either the strain selected for the trial or the fact that the bacteria were nonviable. These studies indicate that there are probably important differences in efficacy between probiotics. It is necessary to think of individual probiotics (like individual antibiotics) rather than to generalize results to the whole class of agents.

Saccharomyces boulardii also had some protective effect in travelers to North Africa and Turkey. Prevention of TD without using systemic antibiotics is highly appealing; further work on probiotics is needed. It must be recognized that all probiotics are not identical and results of studies done with a particular agent should not be generalized to indicate that any probiotic agent would be successful in the same clinical situation (Peltola et al, 1991).

The 2006 guidelines on travel medicine from the Infectious Diseases Society of America recommended the following oral agents for prophylaxis against travelers' diarrhea, even though no antibiotic has been approved for such use by the Food and Drug Administration in the United States: Norfloxacin-400mg once daily Ciprofloxacin$500 \mathrm{mg}$ once daily Rifaximin- $200 \mathrm{mg}$ once or twice daily Bismuth subsalicylate-two tablets chewed four times daily

The guidelines noted that other fluoroquinolones are likely to be effective but have not been studied for prophylaxis and that there is no antibiotic with proven efficacy for prophylaxis against Campylobacter spe- 
cies, which are a more common cause of travelers' diarrhea in south and Southeast Asia.

With respect to bismuth subsalicylate, the doses required are inconvenient for the traveler, and salicylate toxicity is a potential complication.

Vaccination: Administration of a cholera vaccine is not routinely recommended for travelers. However, a number of trials suggest that the oral, killed whole-cell vaccine given with the nontoxic B subunit of cholera toxin (Dukoral) provides protection for travelers against enterotoxigenic E. coli (ETEC) infection. The rationale for such protection is that the B subunit of cholera is antigenically similar to the heat-labile enterotoxin of ETEC. In France, a total of 129 imported cases of cholera were notified between 1973 and 2005 (3.9 cases/y on average). The geographical sources of infection have changed with time: in the 1980 s, $94 \%$ of the patients were infected in Maghreb (Morocco and Algeria) but none were in 2000. On the other hand, Asia and West Africa progressively emerged and now predominate. In spite of certain poorly informed data and possible underdetection, the number of cases of importation appears to be low and falling (Tarantola et al, 2007). The Dukoral vaccine was approved in the United States in late 2006 for use as a travelers' diarrhea vaccine. A conservative estimate that took into account the incidence of ETEC infection throughout the world and the efficacy of the vaccine suggested that it may prevent $\leq 7 \%$ of travelers' diarrhea cases. The 2006 guidelines on travel medicine from the Infectious Diseases Society of America concluded that the decision to use the vaccine to prevent travelers' diarrhea must balance its cost, adverse effects, and limited utility against the known effectiveness and costs of selftreatment (Doron and Gorbach, 2006).

Kabir (2014) reported that cholera vaccines, Shanchol and Dukoral are two-spaceddose oral vaccines comprising large numbers of killed cholera bacteria. The former con- tains Vibrio cholerae $\mathrm{O} 1$ and $\mathrm{O} 139$ cells, and the latter contains $V$. cholerae $\mathrm{O} 1$ cells with the recombinant $\mathrm{B}$ subunit of cholera toxin. In a field trial in Kolkata (India), Shanchol, the preferred vaccine, protected $45 \%$ of the test subjects in all of the age groups and only $17 \%$ of the children under 5 years of age during the first year of surveillance. In a field trial in Peru, two spaced doses of Dukoral offered negative protection in children under 5 years of age and little protection $(15 \%)$ in vaccines over 6 years of age during the first year of surveillance. Little is known about Dukoral's long-term protective efficacy. Both of these vaccines have questionable compositions, using $V$. cholerae O1 strains isolated in 1947 that have been inactivated by heat and formalin treatments that may denature protein. Immunological studies revealed Dukoral's reduced and short-lived efficacy, as measured by several immunological endpoints.

A strategy for vaccination with heat-labile enterotoxin (LT) from enterotoxigenic Escherichia coli (ETEC) via a skin patch may be an effective measure for protection against moderate to severe travelers' diarrhea. This was illustrated in a phase II trial including 201 participants; protection against moderate to severe diarrhea was observed among those randomized to receive $\mathrm{LT}$ patches; protective efficacy $75 \%$ (Campbell and Green, 2006). Alam et al. (2014) stated that multiple infections with diverse enterotoxigenic E. coli (ETEC) strains lead to broad spectrum protection against ETEC diarrhea. They investigated the heat labile toxin B subunit (LTB) and colonization factor antigens (CFA/I and CS6) specific IgA and IgG memory $\mathrm{B}$ cell responses in adult patients and concluded that natural infection with ETEC induces memory B cells and high avidity antibodies to LTB and colonization factor CFA/I and CS6 antigens that could mediate anamnestic responses on re-exposure to ETEC and may help in understanding the requirements to design the effective vaccination strategies. 
Zaidi and Wine (2015) stated that the travelers' diarrhea, affecting millions of travelers every year globally, continues to be a leading cause of morbidity despite advances in vaccination, prevention, and treatment. It will continue to challenge patients and physicians despite the use of sanitation advice, prophylactic vaccines, and treatment with antibiotics. Effects may extend beyond the time of travel, such as post-infectious complications and exacerbation of preexisting disease. Future research should focus on novel strategies for reducing exposure to pathogens, vaccine development, early detection, and targeted treatments.

\section{Conclusion and Recommendations}

The travelers' diarrhea is a common entity caused by a variety of bacteria, viruses, and parasites. It is uncommon to have to make an etiologic diagnosis. Stool cultures or stool examination for ova and parasites should generally be reserved for cases that last beyond 10 to 14 days, except for patients with fever and colitis, those with upper intestinal symptoms in whom giardiasis is more likely, or immunocompromised patients. The mainstay of therapy for travelers' diarrhea is fluid replacement. Attention to fluids including those with sugar and salt is sufficient for mild diarrhea but severe diarrhea should be treated with oral rehydration solution. Packets, which can be reconstituted in clean drinking water, are available for sale in most countries. Travelers should be given a prescription for antibiotics to fill and take with them in case diarrhea develops. Antibiotics should be taken by the traveler if unformed stools occur more than four times a day or for fever, or blood, pus or mucous in stools. Medical care should be sought if fevers persist beyond 10 to 14 days or if fevers become higher or abdominal pain, bloody diarrhea, or vomiting ensue. Anti-motility agents are usually not necessary for mild to moderate diarrhea and should not be used in severe diarrhea except in association with antibiotic therapy. These agents should be discontinued promptly if abdominal pain develops, other symptoms worsen, or diarrhea persists. Attention to choices of food and drink, water purification, and antibiotic prophylaxis are all means of attempting to prevent travelers' diarrhea. Antibiotic prophylaxis is usually reserved for patients in whom dehydration would put them at severe risk.

It should be in mind that the prophylaxis and treatment of populations at risk, children, chronically ill patients, and those on immunosuppressive medications, remain the challenging and require a tailored approach.

\section{References}

Adachi, JA, Ericsson, CD, Jiang, ZD, et al, 2003: Azithromycin found to be comparable to levofloxacin for the treatment of US travelers with acute diarrhea acquired in Mexico. Clin. Infect. Dis. 37:1165.

Adachi, JA, Jiang, ZD, Mathewson, JJ, et al, 2001: Enteroaggregative Escherichia coli as a major etiologic agent in traveler's diarrhea in 3 regions of the world. Clin. Infect. Dis. 32:170612.

Adachi, JA, Mathewson, JJ, Jiang, ZD, et al, 2002: Enteric pathogens in Mexican sauces of popular restaurants in Guadalajara, Mexico, and Houston, Texas. Ann. Int. Med. 136:884-9. Alam, MM, Aktar, A, Afrin, S, Rahman, MA, Aktar, S, 2014: Antigen-specific memory B-cell responses to enterotoxigenic Escherichia coli infection in Bangladeshi adults. PLoS Negl. Trop. Dis. Apr 24;8(4):e2822. doi: 10.1371/journal. pntd.0002822.

Alonso, E, Alfonso, A, Vieytes, MR, Botana, L M, 2015: Evaluation of toxicity equivalent factors of paralytic shellfish poisoning toxins in seven human sodium channels types by an automated high throughput electrophysiology system. Arch. Toxicol. Jan 9. [Epub ahead of print] Avery, ME, Snyder, JD, 1990: Oral therapy for acute diarrhea. N. Engl. J. Med. 323:891.

Bizet, J, Cooper, CJ, Zuckerman, MJ, Torabi, A, Mendoza-Ladd, A, 2014: A bleeding colonic ulcer from invasive Aspergillus infection in an immunocompromised patient: a case report. J Med Case Rep. 5, 8:407.

Briand, V, Buffet, P, Genty, S, et al, 2006: Absence of efficacy of nonviable Lactobacillus acidophilus for the prevention of traveler's diarrhea: a randomized, double-blind, controlled study. Clin. Infect. Dis. 43:1170. 
Burchard, GD, Hentschke, M, Weinke, T, Nothdurft, HD, 2013: Travelers' diarrhea. Dtsch. Med. Wochenschr. 138, 33:1673-83

Campbell, PJ, Green, AR, 2006: The myeloproliferative disorders. N. Engl. J. Med. 355: 2452-9.

CDC, 1971: Shigellosis related to an airplane meal-Northeastern U.S. MMWR 20:397-404.

CDC, 1992: Cholera-international travel, JAM A 268:1648.

Christenson, JC, 2008: Preparing families with children traveling to developing countries. Pediatr. Ann. 37:805-12.

Clemens, JD, Sack, DA, Harris, JR, et al, 1988: Cross-protection by B subunit-whole cell cholera vaccine against diarrhea associated with heat-labile toxin-producing enterotoxigenic $E s$ cherichia coli: results of a large-scale field trial. J. Infect. Dis. 158:372.

DiCesare, D, DuPont, HL, Mathewson, JJ, et al, 2002: A double blind, randomized, placebocontrolled study of SP-303 (Provir) in the symptomatic treatment of acute diarrhea among travelers to Jamaica and Mexico. Am. J. Gastroenterol. 97:2585-92.

Doron, S, Gorbach, SL, 2006: Probiotics: Their role in the treatment and prevention of dis-ease. Expert. Rev. Anti-Infect. Ther. 4, 2:261-75

DuPont, HL, Capsuto, EG, 1996: Persistent diarrhea in travelers. Clin. Infect. Dis. 22:124-9.

DuPont, HL, Hornick, RB, 2003: Adverse effect of Lomotil therapy in shigellosis. JAMA 256:1525.

Dupont, HL, Jiang, ZD, Belkind-Gerson, J, et al, 2007: Treatment of travelers' diarrhea: randomized trial comparing rifaximin, rifaximin plus loperamide, and loperamide alone. Clin. Gastroenterol. Hepatol. 5:451-9.

DuPont, HL, Jiang, ZD, Okhuysen, PC, et al, 2005: A randomized, double-blind, placebo-controlled trial of rifaximin to prevent travelers' diarrhea. Ann. Int. Med. 142:805.

El-Bahnasawy, MM, Aly, NZ, Abdel-Fattah, MA, Morsy, TA, 2014: Botulism as a food poisoning: What is it? J. Egypt. Soc. Parasitol. 44, 1:211-20.

el-Karamany, EM, Zaher, TI, el-Bahnasawy, MM, 2005: Role of water in the transmission of cyclosporiarsis in Sharkia Governorate, Egypt. J. Egypt. Soc. Parasitol. 35:953-60.

Ericsson, CD, Pickering, LK, Sullivan, P, DuPont, HL, 1980: Role of location of food consumption in the prevention of travelers' diarrhea in Mexico. Gastroenterology 79:812.

Golledge, CL, Riley, TV, 1995: Clostridium difficile-associated diarrhea after doxycycline malaria prophylaxis. Lancet 345:1377.

Groh, CD, MacPherson, DW, Groves, DJ, 1996: Effect of heat on the sterilization of artificially contaminated water. J. Travel Med. 3, 1: 11-3.

Hakanen, A, Jousimies-Somer, H, Siitonen, A, et al, 2003: Fluoroquinolone resistance in Campylobacter jejuni isolates in travelers returning to Finland: association of ciprofloxacin resistance to travel destination. Emerg. Infect. Dis. 9:267.

Helms, M, Simonsen, J, Molbak, K, 2006: Foodborne bacterial infection and hospitaliza-tion: A registry-based study. Clin. Infect. Dis. 42:498504.

Hill, DR, Ericsson, CD, Pearson, RD, et al, 2006: The practice of travel medicine: guidelines by the Infectious Diseases Society of America. Clin. Infect. Dis. 43:1499.

Hill, DR, Ericsson, CD, Pearson, RD, et al, 2006: The practice of travel medicine: guidelines by the Infectious Diseases Society of America. Clin. Infect. Dis.43:1499.

Hilton, E, Kolakowski, P, Singer, C, Smith, M, 1997: Efficacy of Lactobacillus GG as a diarrheal preventive in travelers. J. Travel Med. 4: 41.

Huang, DB, Awasthi, M, Le, BM, et al, 2004: The role of diet in the treatment of travelers' diarrhea: A pilot study. Clin. Infect. Dis. 39:468.

Jokipii, L, Pohjola, S, Jokipii, AM, 1985: Cryptosporidiosis associated with traveling and giardiasis. Gastroenterology 89:838.

Kabir, S, 2014: Critical analysis of compositions and protective efficacies of oral killed cholera vaccines. Clin. Vaccine Immunol. 21, 9: 1195-205.

Koo, HL, DuPont, HL, 2010: Rifaximin: a unique gastrointestinal-selective antibiotic for enteric diseases. Curr. Opin. Gastroenterol. 26, 1:17-25.

Liu, W, Liao, L, Shi, H, Wu, B, Li, X, et al, 2014: An analysis of clinical characteristics and risk factors for ulceration in ischemic colitis. Zhonghua Nei Ke Za Zhi. 53, 8:626-30.

Markell, EK, Udkow, MP, 1986: Blastocystis hominis: Pathogen or fellow traveler? Am. J. Trop. Med. Hyg. 35, 5:1023-6 
Mattila, L, Siitonen, A, Kyronseppa, H, 1992: Seasonal variation in etiology of travelers' diarrhea. J. Infect. Dis. 165:385.

Murray, BE, 1986: Resistance of Shigella, Salmonella, and other selected enteric pathogens to antimicrobial agents. Rev. Infect. Dis. 8:S172.

Murray, BE, Mathewson, JJ, DuPont, HL, 1990: Emergence of resistant fecal E. coli in travelers not taking prophylactic antimicrobial agents. Antimicrob. Agents Chemother. 34: 51520.

Musher, DM, Musher, BL, 2004: Contagious acute gastrointestinal infections. N. Engl. J. Med. 351:2417.

Patrício, C, Amaral, P, Lourenço, J, 2014: An uncommon case of hepatopulmonary amoebiasis. BMJ Case Rep. Aug 25; 2014. pii: bcr 2014204129.

Peltola, H, Siitonen, A, Kyronseppa, H, et al, 1991: Prevention of travellers' diarrhoea by oral B-subunit/whole-cell cholera vaccine. Lancet 338:1285.

Petruccelli, BP, Murphy, GS, Sanchez, JL, 1992: Treatment of travelers' diarrhea with ciprofloxacin and loperamide. J. Infect. Dis. 165: 557.

Rendi-Wagner, P, Kollaritsch, H, 2002: Drug prophylaxis for travelers' diarrhea. Clin. Infect. Dis. 34:628.

Riddle, MS, Arnold, S, Tribble, DR, 2008: Effect of adjunctive loperamide in combination with antibiotics on treatment outcomes in traveler's diarrhea: a systematic review and metaanalysis. Clin. Infect. Dis. 47: 1007.

Salam, I, Katerlaris, P, Leigh-Smith, S, Farthing, M, 1994: Randomized trial of single dose ciprofloxacin for traveller's diarrhea. Lancet 344:1537.

Salazar-Lindo, E, Santisteban-Ponce, J, Chea -Woo, E, Gutierrez, M, 2000: Racecadotril in the treatment of acute watery diarrhea in children. N. Engl. J. Med. 343:463.

Sanders, JW, Frenck, RW, Putnam, SD, et al, 2007: Azithromycin and loperamide are comparable to levofloxacin and loperamide for the treatment of traveler's diarrhea in United States military personnel in Turkey. Clin. Infect. Dis. 45:294.

Smith, KE, Besser, JM, Hedberg, CW, et al, 1999: Quinolone-resistant Campylobacter jejuni infections in Minnesota, 1992-1998. N. Engl. J. Med. 340:1525.
Soave, R, Ma, P, 1985: Cryptosporidiosis: Travelers' diarrhea in two families. Ann. Int. Med. 145:70.

Steffen, R, Collard, F, Tornieporth, N, et al, 1999: Epidemiology, etiology, and impact of traveler's diarrhea in Jamaica. JAMA 281:811.

Steffen, R, Sack, DA, Riopel, L, et al, 2003: Therapy of travelers' diarrhea with rifaximin on various continents. Am. J. Gastroenterol. 98: 1073-80.

Steffen, R. 1986: Epidemiologic studies of travelers' diarrhea, severe gastro-intestinal infections and cholera. Rev Infect Dis 1986; 8:S122-9.

Tarantola, A, Ioos, S, Rotureau, B, Paquet, C, Quilici, ML, et al, 2007: Retrospective analysis of the cholera cases imported to France from 1973 to 2005. J. Travel Med. 14, 4:209-14.

Taylor, DN, Sanchez, JL, Candler, W, 1991: Treatment of travelers' diarrhea: Ciprofloxacin plus loperamide compared with ciprofloxacin alone. Ann. Int. Med. 114:731.

Thielman, NM, Guerrant, RL, 2004: Clinical practice: Acute infectious diarrhea. N. Engl. J. Med. 350:38.

To, KB, Napolitano, LM, 2014: Clostridium difficile infection: Update on diagnosis, epidemiology, and treatment strategies. Surg. Infect. (Larchmt). 15, 5:490-502

Tontini, GE, Vecchi, M, Pastorelli, L, Neurath, MF, Neumann, H, 2015: Differential diagnosis in inflammatory bowel disease colitis: State of the art and future perspectives. World J. Gastroenterol. 21, 1:21-46.

Varma, JK, Wu, S, Feng, Z, 2012: Detecting and controlling foodborne infections in hu-mans: lessons for China from the United States experience. Glob Publ. Hlth. 7, 7:766-78.

Von Sonnenburg, F, Tornieporth, N, Waiyaki, P, et al, 2000: Risk and aetiology of diarrhoea at various tourist destinations. Lancet 356 : 133.

Wang, W, Xu, L, 2012: The clinical features and risk factors for 89 cases of ischemic colitis. honghua Nei Ke Za Zhi. 51, 10:769-73

Zaidi, D, Wine, E, 2015: An update on travelers' diarrhea. Curr. Opin Gastroenterol. 31, 1:713. 